Código JEL: M31; I11; I12

\title{
La satisfacción del cliente como indicador de calidad en neurorehabilitación
}

\author{
D. Raúl VILLODRE \\ Universidad Católica de Valencia \\ r.villodre@gmail.com \\ Dra. Remedios CALERO \\ Universidad Católica de Valencia \\ remedios.calero@mail.ucv.es \\ Dra. Martina G. GALLARZA \\ Universitat de València \\ martina.gallarza@uv.es
}

\section{RESUMEN}

Este trabajo pretende conocer el grado de satisfacción del cliente desde una óptica dual: cliente externo (primario en paciente y secundario en familiares) e interno (empleado), como indicador de calidad asistencial en un centro de neurorehabilitación. Los resultados avalan la tendencia de los clínicos a introducir el grado de satisfacción como un índice complementario a las medidas de efectividad y eficiencia para la valoración de los tratamientos. Esto permite coordinar todas las acciones diseñando la actividad asistencial de modo tal que integre el punto de vista del profesional, del paciente y, en el caso de la neurorehabilitación, también el de sus familiares.

Palabras clave: calidad asistencial; satisfacción del cliente; encuestas como metodología.

Sumario: 1. Introducción 2. Marco teórico-conceptual 3. Metodología 4. Resultados 5. Discusión y conclusions. 6. Bibliografía. 


\title{
Customer satisfaction as an indicator of quality in neurorehabilitation service
}

\begin{abstract}
This paper tries to determine customer satisfaction degree from two perspectives: outsider customer (primary in patient and secondary in relatives) and insider customer (employee), being all of them indicators of quality of care in a neurorehabilitation centre. Results support clinician tendency of introducing patient satisfaction degree as an indicator, like effectiveness or efficiency, in order to value success treatment. This tendency allows to coordinate all actions in health care design in a such way that integrates professionals, patients and, in the case of a neurorehabilitation service, relatives perspectives.
\end{abstract}

Keywords: Quality of Health Care; Consumer Satisfaction; Survey Methodology

\section{INTRODUCCIÓN}

Históricamente, la calidad de la relación médico-paciente ha sido considerada como un elemento muy influyente en el resultado de la atención sanitaria. Esta realidad se ha visto acentuada, ya que hemos asistido a un cambio importante en las relaciones médico-paciente como consecuencia de la mayor relevancia que están adquiriendo los consumidores en la sociedad, cada vez más informados y conscientes de sus derechos y opciones de elección (Villegas y Rosa, 2003; Gaminde, 2008). El efecto inmediato es que el resultado de la asistencia sanitaria se mide hoy día en términos de efectividad, eficiencia, percepción por el paciente de su dolor o de su autonomía, su sensación de bienestar físico y mental y, también, por su satisfacción con el resultado alcanzado.

Así, hemos sido testigos en los últimos años de cómo el diseño de los procesos asistenciales ha cambiado sustancialmente cuando los clínicos han empezado a preocuparse por satisfacer no solo las necesidades sanitarias de sus pacientes sino también sus expectativas, relativas tanto al servicio sanitario puro, como a los servicios periféricos que lo rodean. Los pacientes han pasado a ser coproductores de los cuidados de salud y, por tanto, han adoptado un rol diferente, considerándose ahora necesario contar con su opinión y promover en ellos decisiones responsables sobre su salud. El concepto de "cuidados centrados en el paciente", que es el que nos ocupa en este trabajo, obedece a este nuevo planteamiento que parte precisamente de la idea de que las decisiones clínicas deben incluir la perspectiva del paciente (y en ocasiones también la de sus familiares), llamando de este modo la atención sobre el papel activo en la toma de decisiones sobre su salud que los pacientes paulatinamente van adquiriendo (Díaz, 2002; Villegas y Rosa, 2003).

Aunque existe también una cierta controversia en la adopción de este tipo de enfoque, considerado en ocasiones de índole exclusivamente académica y por tanto 
de difícil aplicabilidad (Aibar, 2009), son múltiples las aproximaciones existentes que relatan sus bondades, dentro y fuera de nuestras fronteras (eg. Díaz, 2002; Expósito et al., 2009; Rundle-Thiele y Russell-Bennett, 2010). La novedad de este enfoque consiste en lograr coordinar todas las acciones con el fin de diseñar la actividad asistencial de modo tal que integre el punto de vista tanto del profesional como del paciente. Para alcanzar este objetivo, no basta con obtener unos buenos resultados. En la práctica, observamos que la insatisfacción no proviene siempre del grado de efectividad de las intervenciones (Mira et al, 2000; Escribano, 2005) sino que son muchos otros los factores (subjetivos y circunstanciales) que pueden llegar a determinar la calidad percibida de un acto médico (Cárcamo, 2011). Por esta razón, una gestión eficiente de los recursos sanitarios debe considerar no sólo los aspectos científico-técnicos, sino que, además, debe incidir en otros aspectos más periféricos propios de personal no sanitario, como la organización de las consultas, su accesibilidad, disponibilidad, apariencia física, etc. (Ruiz Moral et al., 2011), así como ser capaz de promover el trabajo en equipo, la creatividad, la cercanía y la motivación de los profesionales (Alonso et al., 2011; Spake y Bishop, 2009).

Como una aportación más, este trabajo tiene por objeto: describir de manera dual (clientes internos y externos) la prestación de un servicio de neurorehabilitación con el interés puesto no sólo en los aspectos médicos, sino también en los periféricos. Esta descripción del nivel de satisfacción de ambos colectivos se entiende como un indicador del nivel de calidad asistencial ofrecida, por lo que se espera permita detectar las debilidades e ineficiencias de los procesos de atención al paciente que, hoy día, son una necesidad de la práctica asistencial no siempre fácil de resolver. La justificación a la realización de este trabajo debemos buscarla en esta necesidad máxima de integración y búsqueda de sinergias entre las percepciones de usuarios (demanda) y prestatarios (oferta) del servicio sanitario, de interés máximo para la gestión sanitaria (Giménez Manzorro et al., 2007; Alonso et al., 2011). A continuación mostramos la provisión de un marco teórico en torno a la noción de satisfacción del cliente, sobre el que engarzamos seguidamente la exposición de la metodología seguida, la obtención de resultados descriptivos mediante medias proyectadas por áreas de la prestación y la discusión de resultados, todo ello referido a un estudio empírico a clientes internos y externos de un servicio integral de neurorehabilitación.

\section{MARCO TEÓRICO-CONCEPTUAL}

La Encuesta de Discapacidad, Autonomía Personal y Situaciones de Dependencia de 2008 (EDAD, INE, 2008) estima en 420.000 las personas afectadas por daño cerebral adquirido en España (9,3 por cada 1.000 habitantes). En España, se diagnostican en torno a 120.000 casos de trombosis cerebral, 
embolia o derrame cerebral al año, calculándose que cada seis minutos una persona sufre uno de estos accidentes cardiovasculares. El 30\% de estos pacientes fallecen y el otro $50 \%$ quedan en situación de dependencia para las actividades de la vida diaria. Como expone Sánchez (2006, p. 31), "el 13\% de las personas con discapacidad en España lo son a causa de las enfermedades neurológicas, que constituyen la primera causa de discapacidad a partir de los 65 años, alrededor del 50\%”. En los próximos años, estas enfermedades afectarán cada vez a un mayor número de personas, sobre todo en países como España, debido al envejecimiento de la población y al aumento de la esperanza de vida por la disminución de la mortalidad de otras enfermedades. Fruto de todo ello, la neurorehabilitación está cobrando un papel cada vez más destacado en el servicio sanitario, motivo por el cual consideramos necesario investigar la calidad asistencial ofrecida medida, en este trabajo, a través de la satisfacción del cliente.

A la luz de la literatura consultada, la satisfacción del cliente se considera un elemento deseable e importante de la actuación médica; no obstante, aunque intuitivamente el concepto es entendido por todos, no existe consenso ni sobre su naturaleza ni sobre su evaluación (Pascoe, 1983; Giese y Cote, 2000). Desde los primeros años la doctrina es coincidente sobre la multidimensionalidad de la noción (Hulka et al., 1970; Wolf et al., 1978; Feletti et al., 1986; Ware y Hays, 1988), pero no sobre el número y naturaleza de estas dimensiones, pudiéndose reconocer de manera genérica y afín con la literatura general sobre satisfacción tres dimensiones: cognitiva (cantidad, tipo y calidad de la información que da el médico); afectiva (interés y comprensión que demuestra); y comportamental (sobre las habilidades y competencias clínicas del médico).

En lo que respecta a su evaluación, siguiendo la línea de investigación de los 80 en marketing de servicios, las fronteras conceptuales y metodológicas entre calidad de servicio y satisfacción son difusas, sobre todo desde que se recurre a la teoría de la "desconfirmación de expectativas" para determinar que la satisfacción es el resultado de la diferencia entre lo que el paciente esperaba que ocurriera y su percepción de la experiencia (Linder-Pelz, 1982), en línea con el reconocido modelo SERVQUAL de Parasuraman et al (1988). Sin embargo, como reconoce la doctrina general sobre evaluación de servicios, esta conceptualización de la calidad presenta problemas técnicos de medición (Siriex y Dubois, 1999; Day y Crasck, 2000), no atiende a la consideración simultánea de otros factores (Woodfruff y Gardial, 1996) como la eficacia de la empresa en la gestión de las expectativas de los clientes, la experiencia de los clientes con productos de la competencia y las opiniones de terceros. A ello debemos sumar la idiosincrasia de los servicios sanitarios que condiciona notablemente la percepción y las expectativas del paciente como usuario del servicio (Cárcamo, 2011). Así, aproximaciones más completas recomiendan el uso de variables mediadoras entre la calidad y la satisfacción, como el valor percibido (Brand et al., 1998) y la inclusión de una última variable de lealtad (comportamental en la reutilización del servicio y/o del 
facultativo, y afectiva en la recomendación) (Spake y Bishop, 2009; Torres et al., 2009; Rundle-Thiele y Russell-Bennett, 2010; Henthorne et al., 2009) para una evaluación más completa de la prestación sanitaria.

En resumen, la satisfacción del paciente está directamente relacionada con el componente afectivo de la actitud hacia el sistema sanitario o cualquiera de sus unidades (consultas o profesionales) y, conceptualmente, puede explicarse merced a la desconfirmación de expectativas, aunque su evaluación por este paradigma sea compleja. Sí resulta evidente que se trata de un concepto multidimensional (Oliver, 1997; Giese y Cote, 2000) cuyos componentes varían en función del tipo de prestación (hospital de agudos, consulta a crónicos, urgencias, etc.). Además de esta realidad común a la evaluación de cualquier servicio, los cambios sociales y tecnológicos acaecidos han definido un espacio de relación diferente según la organización y prestaciones de los diferentes Sistemas de Salud provocando que el rol del paciente no sea el mismo en todos los casos y por ende su participación en el sistema sanitario sea muy variable. En definitiva, los canales por los que un paciente participa en la actualidad en el Sistema Sanitario (Wensing et al., 1994) consistirían en: colaboración activa durante la consulta brindando al médico información fiable sobre su salud; participación en actividades de educación y promoción de la salud; co-responsabilización en la toma de decisiones sobre su salud y en el seguimiento del tratamiento; realización de un uso racional de los recursos sanitarios, aportando sus puntos de vista sobre el funcionamiento del sistema sanitario (su organización y prestaciones); y participación en la evaluación de los resultados que se alcanzan manifestando su satisfacción o insatisfacción con los mismos, tanto desde el punto de vista asistencial como desde otros puntos de vista.

En conclusión, podemos establecer que la medición de la satisfacción de los clientes como indicador de calidad asistencial permite: conocer la percepción de los clientes sobre el servicio que reciben; adaptar el servicio a sus necesidades; orientar el uso de los recursos a la resolución de los problemas más decisivos; y disponer de una información cuantificada para seguir la evolución de los servicios prestados. Para ello, cabe considerar como cliente tanto al externo -paciente- como al interno -empleado- (Swartz y Brown, 1989), puesto que los puntos de vista y las percepciones de uno y otro no siempre coinciden, siendo los médicos normalmente más exigentes consigo mismos respecto a los resultados asistenciales a alcanzar (Wensing y Grol, 1994) y siendo relevante conocer las valoraciones del personal determinantes del clima laboral que redunda en la calidad asistencial prestada (Alonso et al., 2011). Toda esta realidad está en la base del planteamiento de nuestro estudio empírico. 


\section{METODOLOGÍA}

\subsection{La evaluación de la satisfacción en el servicio sanitario}

Bajo el criterio genérico de resultados en los clientes del modelo Europeo de Excelencia (EFQM) que recoge la recomendación de evaluar sistemáticamente la percepción de los clientes externos y recurrir a medidas internas para predecir y mejorar la satisfacción de sus clientes (Arcelay et al, 2000) en la literatura sanitaria, se han analizado las percepciones de los pacientes ante diferentes situaciones (Sutherland et al, 1989); como por ejemplo los factores que provocan insatisfacción con la Atención Primaria (Maderuelo et al, 1996); el trato y la competencia de los médicos (Cromarty, 1996); la compatibilidad de los procesos asistenciales con los deseos de los pacientes (Reiley et al, 1996); o la relación entre expectativas del paciente y su satisfacción (Haas, 1999).

No obstante, el método empleado con mayor frecuencia son las encuestas de satisfacción (Hall y Dornan, 1988; Hulka et al., 1970; Ware y Hays, 1988), la mayoría con una estructura de respuesta tipo Likert que deben contar con el respaldo de los profesionales y de la propia Dirección de los centros para que se deduzcan mejoras sustanciales de sus resultados.

La mayoría de los estudios utilizan cuestionarios de tipo general, como la Escala de Satisfacción con la Entrevista Médica (Medical Interview Satisfaction Scale), Cuestionario de Hulka et al (1970), VSQ (Visit-Specific Satisfaction Questionnaire) de Ware y Snyder (1975), el cuestionario PSQ (Patient Satisfaction Questionnaire) de Ware y Hays (1988), el cuestionario de satisfacción con la atención primaria de Feletti et al (1986), PJHQ56 (Patient Judgements of Hospital Quality) de Meterko et al (1990), la Encuesta de Experiencias del Paciente (Zastowny et al, 1995) y el cuestionario SERVQHOS (Mira et al, 1997) para evaluar la calidad de la asistencia hospitalaria percibida por el paciente.

Asimismo, otros trabajos desarrollan instrumentos de medida de la satisfacción de carácter más específico orientados a contextos o procesos de enfermedad concretos: entre otros, hiperplasia prostática (Barry et al, 1995); quimioterapia (Liu et al, 1997); insuficiencia cardíaca (Candlish et al, 1998); reumáticos (Sixma et al, 1998); o usuarios de servicios como mamografía (Loeken et al, 1997); atención continuada en primaria (McKinley et al, 1997); o consentimiento informado (Sáinz et al, 1997). Desde nuestro conocimiento, ningún trabajo anterior se ha interesado por el servicio de neurorehabilitación.

\subsection{Descripción de la metodología seguida en el estudio empírico}

Como una aportación más en esta línea de medir la especificidad de un servicio sanitario y no su generalidad, el presente trabajo, como se ha señalado anteriormente, pretende conocer el grado de satisfacción del cliente, tanto externo como interno, como indicador de la calidad asistencial en un contexto específico, en este caso en un centro especializado en neurorehabilitación. A tal efecto, se ha 
elegido un Instituto especializado en la atención a personas que han sufrido una alteración de orden neurológico. El centro, situado en la ciudad de Valencia, posee $1.500 \mathrm{~m} 2$ de instalaciones dedicadas a la valoración, rehabilitación, formación e investigación del daño cerebral sobrevenido. Dada la complejidad del tratamiento del daño cerebral adquirido, la rehabilitación se plantea desde un equipo multidisciplinar cubriendo todas las necesidades no solo del paciente sino también de la familia y/o del cuidador habitual.

A partir de la revisión de la literatura realizada y considerando las características particulares del centro, se ha diseñado y realizado un análisis cuantitativo consistente en dos encuestas de satisfacción dirigidas una al cliente interno y otra al externo. A tenor de la diversidad de medidas y evaluaciones de calidad y satisfacción señaladas más arriba, y teniendo en cuenta la especificidad del servicio sanitario que como investigadores deseemos evaluar, se ha optado por la confección de un cuestionario ad-hoc, en donde se incluyeron indicadores de elaboración propia siguiendo la distinción clásica de servicios centrales y periféricos, pero adaptados a las necesidades de un centro de neurorehabilitación. El objetivo con el que se confeccionaron las encuestas consistía, en una primera fase, en identificar el nivel de satisfacción de los clientes y conocer los puntos fuertes y débiles de los servicios prestados para, en una segunda fase, poner en marcha un plan de mejora. Las áreas seleccionadas para el cuestionario dirigido al cliente externo fueron aquellas habitualmente consideradas a efectos administrativos del centro, distinguiendo entre servicios centrales y periféricos. Así, se estimaron en cuatro: información telefónica, acogida en las instalaciones, proceso de rehabilitación y procesos administrativos, añadiendo una categoría final de comentarios relativa al nivel general de satisfacción y predisposición a la recomendación o lealtad afectiva.

Respecto al cuestionario dirigido al cliente interno, se incluyeron cuestiones relativas a la relación entre el empleado y el centro, el espíritu de equipo, la relación con la dirección, información y comunicación, su percepción de la calidad otorgada a los clientes, su formación como empleados y sus perspectivas de futuro. Los cuestionarios resultantes recogían en cada bloque una serie de cuestiones (entre 4 y 15 por cada área) a valorar en función de una escala Likert de cuatro puntos. En relación a la forma de administración del cuestionario, ambos cuestionarios se entregaron en el centro y se habilitó un buzón en el que tanto los pacientes como los empleados depositaron sus respuestas manteniendo el anonimato.

La población objetivo de la encuesta fue la totalidad de clientes atendidos en el centro, concretamente 35 clientes adultos y 37 niños, así como los 17 empleados que constituían la plantilla. La muestra recogida y analizada fue de 17 clientes internos y 37 clientes externos, siendo el índice de respuesta del $45 \%$ de los pacientes adultos, del 57\% en el caso de la población infantil (cuestionario que fue cumplimentado en su mayoría por familiares, dadas las características de esta población) y del 100\% para el caso de los empleados. 


\section{RESULTADOS}

Las encuestas realizadas han permitido conocer la opinión de los clientes, tanto externos como internos, obteniendo del análisis cuantitativo los resultados que mostramos a continuación. Puesto que el enfoque del trabajo es meramente descriptivo, el análisis estadístico seguido fue la realización de medias aritméticas; estas medias se proyectaron en forma de estrellas según las áreas evaluadas, para una mejor visualización de estos resultados.

Figura 1. Valoración del cliente externo por áreas.
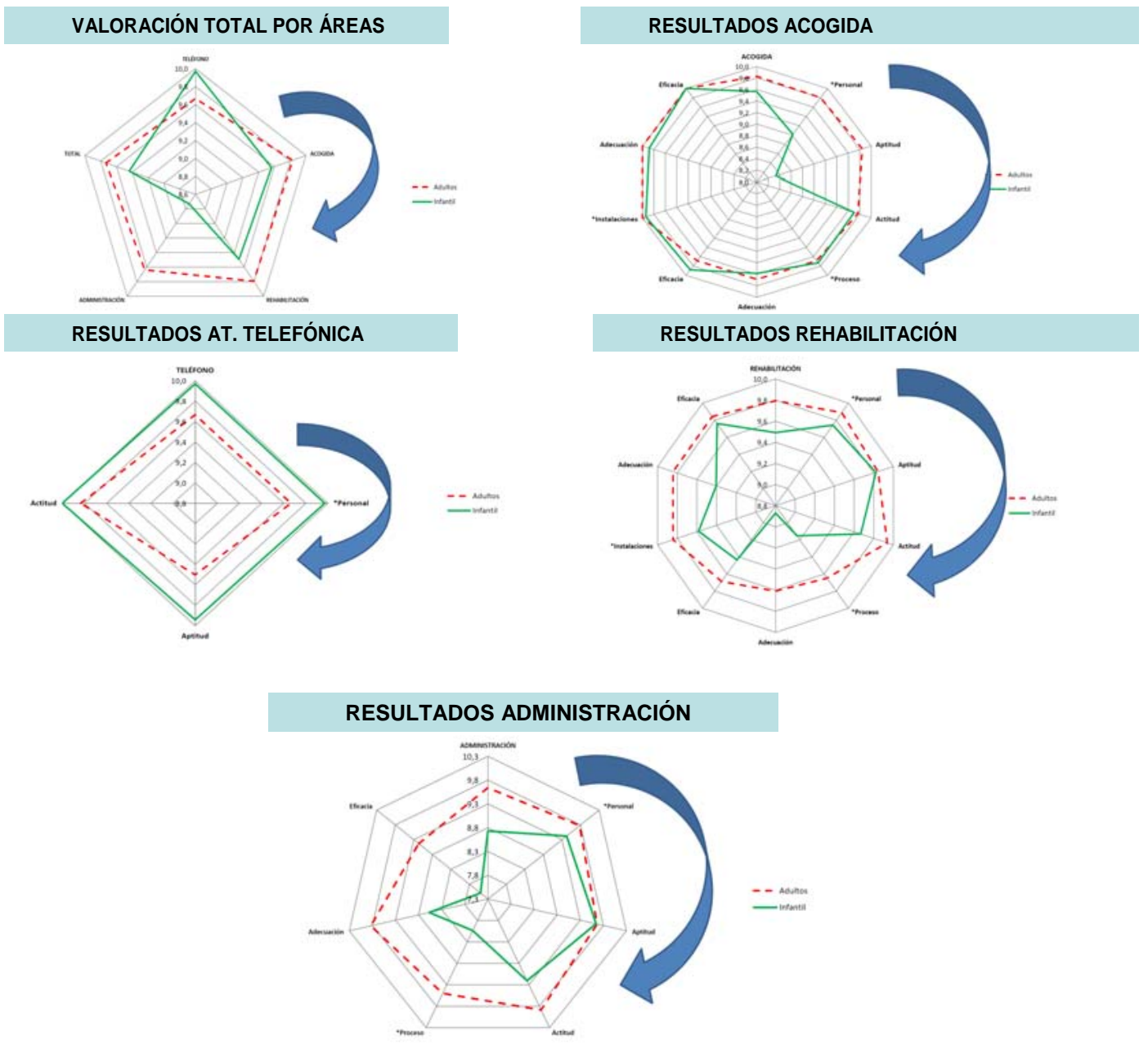

Fuente: Elaboración propia 
Centrándonos en primer lugar en el cliente externo (Figura 1), los pacientes se muestran satisfechos con el método de rehabilitación, su eficacia y el nivel de información. La valoración general de las distintas unidades, pese a ser superior en adultos que en pediatría, están muy próximas. La mayor diferencia se encuentra en los procesos de acogida y atención telefónica. Respecto al área de rehabilitación, se ha detectado que el nivel de información sobre la evolución del paciente, aún siendo bueno, es inferior en pediatría que en adultos. Se debería, por tanto, dar a conocer de una manera más adecuada cuáles son los principios del método de rehabilitación de esta institución, sobre todo en pediatría. Asimismo, se han detectado otras dos áreas de mejora: la capacidad de transmitir tranquilidad ante las complicaciones y la necesidad de ofrecer más ayuda para tratar los problemas con la Administración. Esta es una evidencia de la particular complejidad del servicio sanitario, que conceptualmente es reconocido como de los más críticos (Calero y Gallarza, 2010) ya que supone una mayor indefensión en el consumidor que en ningún otro servicio.

En cuanto al equipo humano, resulta muy bien valorado, destacando su amabilidad en el trato, la confianza que percibe el paciente y el interés que tiene en su evolución. El componente humano, también reconocido como crucial en el servicio sanitario (Navarro Espigares y Hernández Torres, 2011; Henthorne et al., 2009) emerge en este estudio con fuerza y con una alta valoración.

En las encuestas realizadas, se reservó un espacio abierto para que los pacientes expresaran aquellos comentarios que considerasen oportunos, revelándose las siguientes necesidades: nuevas técnicas de rehabilitación, construcción de una piscina y mejoras en la temperatura de la unidad infantil. Resulta relevante la complejidad de los distintos agentes implicados en el servicio sanitario, los familiares como prescriptores del servicio son públicos diferentes de los que es determinante conocer su grado de satisfacción, máxime en una atención sanitaria como la neurorehabilitación. En nuestro caso, las encuestas en su mayoría fueron rellenadas por los familiares valorando, tanto la atención recibida por parte de su familiar como la incidencia de sus propias expectativas del servicio recibido.

Respecto al cliente interno o empleados (cf. Figura 2), se evaluaron ocho ítems: imagen; cambios; espíritu de grupo; dirección; formación; información y comunicación; clientes; y futuro. A la luz de los resultados, se detecta buen clima de trabajo, espíritu de equipo y gran confianza en el futuro del centro. Así, se han detectado como fortalezas: buena relación entre los distintos servicios (agregabilidad del servicio sanitario); espíritu de equipo muy consolidado; los empleados cuentan con información suficiente del trabajo que deben realizar; apreciación de los cambios producidos en la organización; confianza en el futuro; y valoración muy positiva de la oportunidad de trabajar en esta institución. En lo relativo a debilidades, se ha puesto de manifiesto: necesidad de mejora del estilo de dirección; escasa satisfacción con el nivel salarial; falta de concreción de los 
objetivos; e insatisfacción con la formación recibida, en especial la relativa a calidad.

Figura 2. Valoración del cliente interno.

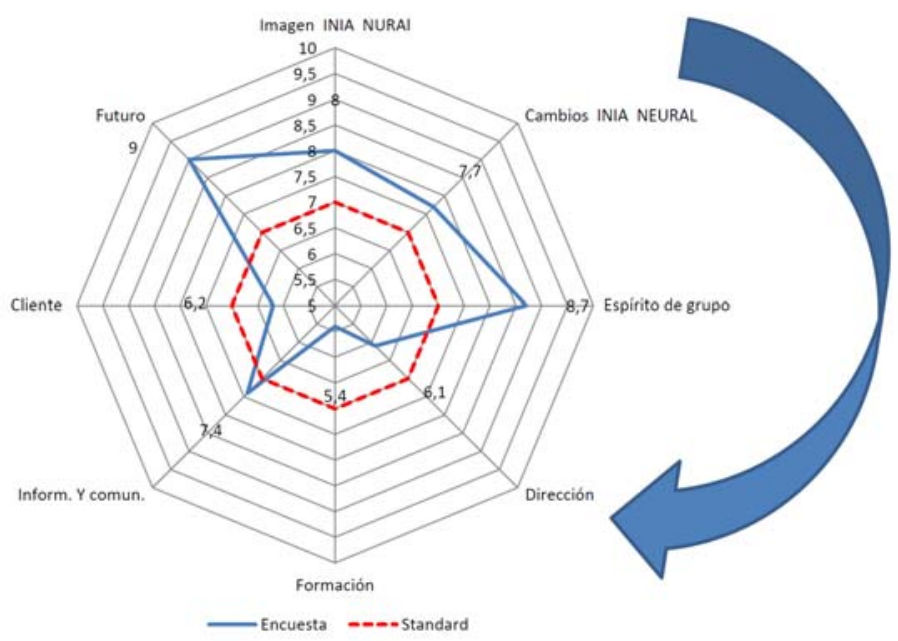

Fuente: Elaboración propia.

Todos estos resultados permiten establecer áreas de mejora y consecuentemente planes de acción para la dirección, como una posibilidad de monitorización de la calidad asistencial ofrecida a los usuarios, además de servir como sistema de retroalimentación a la hora de modificar algunas conductas de la organización. De manera adicional, en línea con el objetivo del trabajo a partir de la información obtenida en dichas encuestas se propusieron cinco planes de acción, distinguiendo para cada uno de los planes, objetivos, indicadores, periodicidad y responsables (cf. Cuadro 1). 
Cuadro 1. Planes de acción implantados a partir de la información obtenida en las encuestas de satisfacción

\begin{tabular}{|c|c|c|c|c|c|c|c|}
\hline & PROCESO & $\begin{array}{c}\text { CONCLUSIÓN DE LA } \\
\text { ENCUESTA }\end{array}$ & OBJETIVO & PLAN DE ACCIÓN & INDICADORES & PERIODICIDAD & RESPONSABLE \\
\hline \multirow{2}{*}{$\begin{array}{l}\text { PLAN DE } \\
\text { ACCIÓN } 1\end{array}$} & \multirow{2}{*}{ Rehabilitación } & \multirow{2}{*}{$\begin{array}{l}\text { El nivel de información sobre la } \\
\text { evolución del paciente, siendo } \\
\text { bueno, es inferior en Infantil } \\
\text { comparado con el de Adultos } \\
\text { (pregunta 22) }\end{array}$} & \multirow{2}{*}{$\begin{array}{l}\text { Asegurar el flujo de información } \\
\text { sobre la evolución de los } \\
\text { pacientes a los familiares }\end{array}$} & \multirow{2}{*}{$\begin{array}{l}\text { Establecer un proceso de información } \\
\text { periódica, favoreciendo la entrada de } \\
\text { los familiares a las sesiones y } \\
\text { facilitando el contacto con las sesiones } \\
\text { de rehabilitación y su evolución }\end{array}$} & $\begin{array}{l}\text { Reclamaciones } \\
\text { atendidas }\end{array}$ & Mensual & \multirow{2}{*}{ Director Técnico } \\
\hline & & & & & $\begin{array}{l}\text { Encuesta de } \\
\text { satisfacción }\end{array}$ & Semestral & \\
\hline $\begin{array}{l}\text { PLAN DE } \\
\text { ACCIÓN } 2\end{array}$ & Rehabilitación & $\begin{array}{l}\text { Necesidad de dar a conocer } \\
\text { mejor cuáles son los principios } \\
\text { del método de rehabilitación de } \\
\text { la Institución, sobre todo en el } \\
\text { área de pediatría (preguntas } 23 \\
\text { y 24) }\end{array}$ & $\begin{array}{l}\text { Mejorar el nivel de información } \\
\text { tanto escrita como oral que los } \\
\text { familiares y pacientes reciben } \\
\text { del responsable de cada unidad } \\
\text { en el proceso de acogida }\end{array}$ & $\begin{array}{l}\text { Revisar el dossier informativo que se } \\
\text { entrega en la primera visita del proceso } \\
\text { de rehabilitación y trabajar con los } \\
\text { responsables de cada unidad la } \\
\text { comunicación del mismo a los } \\
\text { familiares }\end{array}$ & $\begin{array}{l}\text { Encuesta de } \\
\text { satisfacción }\end{array}$ & Semestral & Director Técnico \\
\hline \multirow{2}{*}{$\begin{array}{l}\text { PLAN DE } \\
\text { ACCIÓN } 3\end{array}$} & \multirow[t]{2}{*}{ Rehabilitación } & \multirow{2}{*}{$\begin{array}{l}\text { Solicitud de nuevas técnicas de } \\
\text { rehabilitación (comentarios } \\
\text { proceso de rehabilitación) }\end{array}$} & \multirow{2}{*}{$\begin{array}{l}\text { Ampliar la oferta asistencial con } \\
\text { terapias innovadoras y seguras } \\
\text { que mejoren los resultados en } \\
\text { rehabilitación }\end{array}$} & $\begin{array}{l}\text { Incorporar la realidad virtual como } \\
\text { técnica para conseguir objetivos } \\
\text { funcionales; adquirir más ordenadores } \\
\text { y software especializado; adquirir una } \\
\text { plataforma de valoración del equilibrio }\end{array}$ & $\begin{array}{l}\text { Discapacidad y } \\
\text { dependencia }\end{array}$ & Semestral & \multirow[t]{2}{*}{ Director Técnico } \\
\hline & & & & $\begin{array}{l}\text { y la marcha; desarrollar una mesa de } \\
\text { trabajo táctil en colaboración con la } \\
\text { Universidad que permita controlar } \\
\text { variables en los ejercicios propuestos } \\
\text { en neuropsicología y logopedia }\end{array}$ & $\begin{array}{l}\text { Encuesta de } \\
\text { satisfacción }\end{array}$ & Semestral & \\
\hline $\begin{array}{l}\text { PLAN DE } \\
\text { ACCIÓN } 4\end{array}$ & Administración & $\begin{array}{l}\text { Escasa información a la hora de } \\
\text { resolver problemas con la } \\
\text { Administración (preguntas } 32 \text { y } \\
\text { 33) }\end{array}$ & $\begin{array}{l}\text { Facilitar los trámites con la } \\
\text { Administración y resolver los } \\
\text { problemas que puedan surgir }\end{array}$ & $\begin{array}{l}\text { Especializar en trámites administrativos } \\
\text { a una persona que tenga relación } \\
\text { directa y continua tanto con los } \\
\text { familiares como con la Administración }\end{array}$ & $\begin{array}{l}\text { Encuesta de } \\
\text { satisfacción }\end{array}$ & Semestral & $\begin{array}{l}\text { Responsable de } \\
\text { administración }\end{array}$ \\
\hline $\begin{array}{l}\text { PLAN DE } \\
\text { ACCIÓN } 5\end{array}$ & Elementos & $\begin{array}{l}\text { Necesidad de una piscina } \\
\text { (comentarios acogida en las } \\
\text { instalaciones) }\end{array}$ & $\begin{array}{l}\text { Valorar la posibilidad de tener } \\
\text { piscina en las instalaciones }\end{array}$ & $\begin{array}{l}\text { Una vez valorado de forma positiva por } \\
\text { el director técnico responsable de la } \\
\text { calidad asistencial y por el } \\
\text { departamento económico, se prioriza la } \\
\text { creación de una piscina para apoyar el } \\
\text { tratamiento con hidroterapia }\end{array}$ & \multicolumn{3}{|c|}{$\begin{array}{l}\text { SEGUIMIENTO: Hoy en día, la Institución cuenta con } \\
\text { piscina terapéutica, contando con autorización de la } \\
\text { a Administración para disponer de hidroterapia como } \\
\text { I parte de la terapia multidisciplinar }\end{array}$} \\
\hline
\end{tabular}

Fuente: Elaboración propia.

Por todo ello, en base a los resultados de la encuesta de satisfacción, observamos que: se ha conocido la percepción de los clientes internos y externos; se han establecido planes de acción para adaptar el servicio a sus necesidades y orientar el uso de los recursos a la resolución de los problemas; y se ha obtenido una información cuantificada que permitirá seguir la evolución de los servicios prestados en un futuro. En definitiva, se confirma cómo la medición de la satisfacción de los clientes resulta un indicador de calidad asistencial en un centro de neurorehabilitación.

\section{DISCUSIÓN Y CONCLUSIONES}

En este trabajo hemos revisado y analizado la satisfacción con un servicio sanitario específico desde una doble perspectiva: clientes internos y externos. Para ello, entendemos la satisfacción del cliente como un indicador del resultado de la atención médica, como propuesta de una nueva estrategia de organización y diseño de los procesos asistenciales que pone su énfasis en que dichos procesos deben concebirse considerando tanto las necesidades como la satisfacción de las expectativas de los pacientes. De la revisión de la literatura efectuada se desprende que los estudios sobre esta medida aparecen en forma dispersa sin que se haya 
sistematizado la investigación a este respecto. No obstante, sí resulta indudable cómo la importancia que la medida de la satisfacción ha adquirido en la gestión sanitaria es cada día más notoria.

A tenor de los resultados obtenidos, podemos afirmar en primer lugar la multidimensionalidad de la satisfacción del paciente, en la medida en que son varias las realidades evaluadas con resultados no siempre coincidentes. Resulta imperativo evaluar diferentes aspectos de la prestación sanitaria (informativos, de procesos, técnicos, sociales...) a la vez que comprender que el paciente es parte y juez de la prestación, puesto que es capaz de evaluar la calidad médica de la asistencia recibida. Nuestro estudio apunta no sólo hacia la importancia de los aspectos comunicacionales de gestión de la información, sumados a los aspectos técnicos (todos ellos de carácter cognitivo), sino que incide también en la necesidad de contemplar aspectos afectivos de la asistencia del personal, como la empatía, el apoyo o la amabilidad. En ese sentido, nuestro estudio apunta en la dirección de las diferencias que la literatura señala entre calidad y satisfacción: la primera es de carácter cognitivo, y la segunda cognitivo-afectiva (Giese y Cote, 2000); esta dualidad se hace imperativa en la evaluación del servicio sanitario, mejor aprehendido por tanto desde una óptica cognitivo-afectiva que desde una óptica exclusivamente cognitiva.

En segundo lugar, destacamos el valor del uso de la información en un sentido doble; por un lado se detecta la necesidad de informar más y mejor a los pacientes y a los familiares, lo que como la literatura indica no siempre resulta obvio para el prestador del servicio (Aibar, 2009). Por otro lado, es destacable el uso que los pacientes hacen de la información que reciben basando una parte importante de su decisión sobre dónde buscar ayuda médica, precisamente, en la información sobre el nivel de satisfacción de otros pacientes atendidos por esos médicos, resultado éste en consonancia con otros estudios recientes en otros países (Henthorne et al., 2009). Así, de manera consistente con otros trabajos, se pone de manifiesto la existencia de un mecanismo ya conocido de lealtad afectiva vía recomendación que se hace presente de manera muy significativa en el caso del servicio sanitario (Calero y Gallarza, 2010).

En definitiva, se confirma cómo el clínico interesado en mejorar la prestación de su servicio no debe perder de vista que es recomendable explorar con mayor detalle las relaciones de la satisfacción con el estatus de salud alcanzado por el paciente tras un tratamiento y con el nivel de calidad de vida del paciente, pulsando también la opinión del paciente sobre los servicios periféricos como la provisión de información, la atención telefónica o la cortesía y amabilidad de los sanitarios. Creemos además que es importante la denominación de cliente sin las connotaciones mercantilistas que conlleva, pues el cliente es el que elige, si no le gusta cambia, es preguntado sobre las decisiones e interviene en el diseño asistencial. Bien es cierto que todas estas afirmaciones deben hacerse a la lectura de lo que se entiende por un cliente-paciente cada vez más competente y 
consecuente (Aibar, 2009). Es esta doble responsabilidad paciente-clínico la que enriquece la prestación sanitaria y da lugar a lo que la literatura más reciente sobre marketing de servicios conoce como procesos de co-creación de valor, que creemos son claves para una comprensión mejor de la particularidad del interface médico/paciente del servicio sanitario.

Una tercera línea de actuación que también destacamos de este trabajo se refiere a que no sólo son clientes los pacientes. No podemos olvidar que también lo son, aunque en diferente medida y según el servicio, los familiares de los pacientes: tienen sus expectativas de la atención recibida y debemos darle la importancia que tienen como prescriptores del servicio, teniendo en cuenta el qué, cómo y cuánta información reciben, su formación en medidas preventivas y cuidados a su familiar, la gestión de los tiempos de espera, etc.

La utilidad de este trabajo debemos buscarla en dos realidades: el ámbito elegido y los públicos investigados. Una clínica que presta un servicio de neurorehabilitación es un ejemplo de máximo interés, no sólo por el peso que está adquiriendo esta especialidad en el ámbito sanitario, sino también por la dificultad de tratar con personas que han sufrido alguna alteración neurológica. Si esta información es combinada, además, con la medida de la satisfacción del prestador del servicio (empleados) se consigue una foto más completa y precisa de la calidad asistencial conseguida. Este estudio ofrece, pues, la particularidad de contar con las percepciones tanto de pacientes y/o familiares de pacientes (clientes primarios y secundarios) como de los empleados (clientes internos).

Al término de este trabajo, resulta necesario nombrar algunas limitaciones, tanto de orden teórico-conceptual como metodológico. Las primeras se refieren esencialmente a la difícil delimitación existente entre las nociones de calidad de servicio y de satisfacción, aspecto sobre el que este estudio no se ha detenido, aún siendo los autores conocedores de que el tema ha preocupado a la doctrina durante años (e.g. Giese y Cote, 2000; Siriex y Dubois, 1999). El servicio sanitario parece ser paradigmático para reflexionar y analizar tales diferencias, lo que apuntamos como posibles futuras orientaciones de nuestro trabajo. Las segundas limitaciones, de orden metodológico, se refieren al tipo de muestreo (de conveniencia), al tamaño muestral (reducido) y al tipo de análisis desarrollado (descriptivo). En ulteriores análisis, serían necesarias pruebas de fiabilidad y validez de las escalas utilizadas, si se opta por ejemplo por planteamientos causales de relaciones entre la satisfacción y la lealtad entre otros. La distinción entre cliente primario (paciente) y secundario (familiar), aunque en el origen del planeamiento de este trabajo ha sido insuficientemente explorada debido a las características propias del colectivo investigado en futuras réplicas se debería profundizar en esta distinción ampliando el marco muestral y proponiendo tratamientos multivariantes para conocer mejor la experiencia de un servicio tan particular y rico como el investigado. 


\section{BIBLIOGRAFÍA}

AIBAR, C. (2009). “¿Nos creemos de verdad la necesidad de participación del paciente?”, Revista de Calidad Asistencial, Vol. 24, no 5, p. 183-184.

ALONSO, E, RUBIO, A., MARCH, JC y DANET, A. (2011). "Clima emocional y comunicación interna en una unidad de gestión clínica y dos servicios hospitalarios tradicionales”, Revista de Calidad Asistencial, Vol. 26, no 5, p. 281-284.

ARCELAY, A, LORENZO, S, BACIGALUPE, M y MIRA, JJ. (2000). "Adaptación de un modelo de Calidad Total al sector sanitario", Revista de Calidad Asistencial, Vol. 15, p. 184-191.

BARRY, MJ, FOWLER FJ, MULLEY, AG, HENDERSON, JV y WENNBERG, JE. (1995). "Patient reactions to a program designed to facilite patient participation in treatment decisions for benign prostatic hyperplasia”, Medical Care, Vol. 33, p. 771-782.

BRAND, RR, CRONIN, JJ y ROUTLEDGE, JB. (1998). "Marketing to older patients", Health Marketing Quarterly, Vol. 15, no 2, p. 1-31.

CALERO, R y GALLARZA, MG. (2010). "Marketing de servicios: análisis aplicado a la concesión sanitaria administrativa”, Revista Española de Economía de la Salud, Vol. 9, p. 22-27.

CANDLISH, P, WATTS, P, REDMAN, S, WHYTE, P y LOWE, J. (1998). "Pacientes de edad avanzada con insuficiencia cardíaca: un estudio sobre la satisfacción con la atención recibida y con la calidad de vida”, Revista de Calidad Asistencial, Vol. 13, p. 408-412.

CÁRCAMO, CR. (2011). “Calidad percibida: ¿ilusión o percepción?”, Revista de Calidad Asistencial, Vol. 26, no 3, p.184-187.

CROMARTY, I. (1996). "What do patients think about during their consultations? A qualitative study”, British Journal of General Practice, Vol. 46, p. 525-528.

DAY, E y CRASK, MR. (2000). "Value assessment: the antecedent of customer satisfaction", Journal of Consumer Satisfaction, Dissatisfaction and Complaining Behavior, Vol.13, p. 42-50.

DIAZ, R. (2002). "Satisfacción del paciente: principal motor y centro de los servicios sanitarios”, Revista de Calidad Asistencial, Vol. 17, no 1, p. 22-29.

Encuesta de Discapacidad, Autonomía Personal y Situaciones de Dependencia (2008). Instituto Nacional de Estadística, Ministerio de Sanidad y Política Social.

ESCRIBANO, O. (2005). Gestión clínica en cirugía. Madrid: Arán Ediciones. 633 p. ISBN: 84-959-1361-5.

EXPÓSITO, JA, MARTÍNEZ-SAHUQUILLO, ME y ECHEVARRIA, C. (2009). "Indicadores de calidad asistencial en rehabilitación”, Rehabilitación, Vol. 43, no 3, p. 131-137. 
FELETTI, G, FIRMAN, D y SANSON-FISHER, R. (1986). "Patient satisfaction with primary-care consultations”, Journal of Behavioral Medicine, Vol. 9, p. 389-399.

GAMINDE, I. (2008). "La participación de los pacientes", Revista de Calidad Asistencial, Vol. 23, no 3, p. 93-94.

GIESE, JL y COTE, JA. (2000). "Defining consumer satisfaction”, Academy of Marketing Science Review, Vol. 1, p. 1-34.

GIMÉNEZ MANZORRO, A, VIGIL, D, DURÁN, ME, MARTÍNEZ FERNÁNDEZ-LLAMAZARES, C, LOBATO, E, CARO, L y SANJURJO, M. (2008). "Encuestas de satisfacción al personal facultativo y de enfermería como método de mejora de la calidad de los procesos del servicio de farmacia”, Revista de Calidad Asistencial, Vol. 23, no 1, p. 3-6.

HAAS, M. (1999). "The relationship between expectations and satisfaction: a qualitative study of patients' experiences of surgery for gynaecological cancer”, Health Expectations, Vol. 2, p. 51-60.

HALL, J y DOMAN, M. (1988). "What patients like about their medical care and how often they are asked: a meta-analysis of the satisfaction literature", Social Science \& Medicine, Vol. 27, p. 935-939.

HENTHOME, TL, SALGAONKAR, P y GEORGE, BP. (2009). "External recommendations versus internal satisfaction in health care: a case study in India”, Health Marketing Quarterly, Vol. 26, no 3, p. 241-250.

HULKA, B, ZYZANSI, S, CASSEL, J y THOMPSON, S. (1970). "Scale for the measurement of attitudes toward physicians and primary medical care", Medical Care, Vol. 8, p. 429-435.

LINSER-PELZ, S. (1982). "Toward a theory of patient satisfaction", Social Science \& Medicine, Vol. 16, p. 577-582.

LIU, G, FRANSSEN, E, FITCH, M y WARMER, E. (1997). "Patient preferences for oral versus intravenous palliative chemotherapy", Journal of Clinical Oncology, Vol. 15, p. 110-115.

LOEKEN, K, STEINE, S, SANDVIK, L y LAERUM, E. (1997). “A new instrument to measure patient satisfaction with mammography", Medical Care, Vol. 35, p. 731-741.

MADERUELO, JA, CARRIEDO, E, SERRANO, M, ALMARAZ, A y BERJÓN, AC. (1986). "Factores de insatisfacción con la asistencia sanitaria. Un estudio Delphi”, Atención Primaria, Vol. 17, p. 80-84.

MCKINLEY, RK, MANKU-SCOTT, T, HASTINGS, AM, FRENCH, DP y BAKER, R. (1997). "Reliability and validity of a new measure of patient satisfaction with out of ours primary medical care in the United Kingdom: development of a patient questionnaire”, British Medical Journal, Vol. 314, p. 193-198.

METERKO, M, NELSON, E, RUBIN, H, BATALDEN, P, BERWICK, D y HAYS, R. (1990). "Ware Patients' judgements of hospital quality: report of a pilot study”, Medical Care, Vol. 9, p. S1-S56. 
MIRA, JJ, BUIL, JA, LORENZO, S, VITALLER, J y ARANAZ, J. (2000). "Marketing sanitario y calidad asistencial: reflexiones para el diseño de los servicios quirúrgicos”, Cirugía Española, Vol. 67, p. 180-183.

MIRA, JJ, ARANAZ, J, RODRÍGUEZ-MARÍN, J, BUIL, JA, CASTELL, M y VITALLER, J. (1997). "SERVQHOS: un cuestionario para evaluar la calidad percibida de la asistencia hospitalaria”, Medicina Preventiva, Vol. IV, p. 1218.

NAVARRO ESPIGARES, JL y HERNÁNDEZ TORRES, E. (2011). "Efficiency and quality in health services: a crucial link”, The services industries journal, Vol. 31, p. 385-403.

OLIVER, RL. (1997). Satisfaction. A behavioral perspective on the consumer. New York: McGraw Hill, 521 p. ISBN: 978-0-7656-1770-5.

PARASURAMAN, A, ZEITHMAL, VA y BERRY, LL. (1988). Calidad total en la gestión de servicios. Madrid: Ed. Díaz de Santos, 272 p. ISBN: 84-7978061-4.

PASCOE, G. (1983). "Patient satisfaction in primary health care: a literature review and analysis", Evaluation Program Plan Special Issue, Vol. 6, p. 185418.

REILEY, P, PIKE, A, PHIPPS, M, WEINER, M y MILLER, N. (1996). "Stengrevics S, et al. Learning from patients: a discharge planning improvement project”, Journal on Quality Improvement, Vol. 22, p. 311-322.

RUIZ MORAL, R, ALBA, A, JIMÉNEZ, C, GONZÁLEZ NEUBAUER, V, GARCÍA TORRES, M, PÉRULA, LA y BARRIOS, L. (2011). "Percepción de los pacientes sobre la atención recibida por el personal no sanitario en los centros de salud”, Revista de Calidad Asistencial, Vol. 26, no 2, p. 97-103.

RUNDLE-THIELE, S y RUSSELLl-BENENETT, R. (2010). "Patient influences on satisfaction and loyalty for GP services”, Health Marketing Quarterly, Vol. 27, no 2, p. 195-214.

SÁINZ, A, MARTÍNEZ, B, QUINTANA, O, FERNÁNDEZ, A, FERREIRA, A, NARTÍN, D, et al. (1997). "Consentimiento informado y satisfacción de los pacientes: resultados de una encuesta telefónica realizada a pacientes de 5 hospitales del Insalud”, Revista de Calidad Asistencial, Vol. 12, p. 100-106.

SÁNCHEZ, C. (2006). "Impacto sociosanitario de las enfermedades neurológicas en España”, Fundación Española de Enfermedades Neurológicas.

SIRIEX, L y DUBOIS, PL. (1999). "Vers un modèle qualité-satisfaction integrant la confiance?”, Recherche et Applications Marketing, Vol. 14, p. 1-22.

SIXMA, HJ, KERSENNS, J, VAN CAMPEN, C y PETERS, L. (1988). "Quality of care from the patients perspective: from theoretical concept to a new measuring instrument”, Health Expectations, Vol. 1, p. 82-95.

SPAKE, DF y BISHOP, JS. (2009). "The impact of perceived closeness on the differing roles of satisfaction, trust, commitment, and comfort on intention to remain with a physician”, Health Marketing Quarterly, Vol. 26, no 1, p. 1-15. 
SUTHERLAND, HJ, LOCKWOOD, GA, MINKIN, S, TRITCHLER, DL, TILL, JE, y LLEWELLYN-THOMAS, HA. (1989). "Measuring satisfaction with health care: A comparison of single with paired rating strategies", Social Science \& Medicine, Vol. 28, p. 53-58.

SWARTZ, T y BROWN, S. (1989). "Consumer and provider expectations and experiences in evaluating professional service quality", Journal of the Academy of Marketing Science, Vol. 17, p. 189-195.

TORRES, E, VASQUEZ-PARRAGA, AZ y BARRA, C. (2009). "The path of patient loyalty and the role of doctor reputation”, Health Marketing Quarterly, Vol. 26, p. 183-197.

VILLEGAS, MM y ROSA, IM. (2003). "La calidad asistencial: concepto y medida”, Dirección y Organización, Vol. 29, p. 50-58.

WARE, J y HAYS, R. (1988). "Methods for measuring patient satisfaction with specific medical encounters”, Medical Care, Vol. 26, p. 393-402.

WARE, J y SNYDER, M. (1975). "Dimensions of patient attitudes regarding doctors and medical care services”, Medical Care, Vol. 13, p. 669-682.

WENSING, M, GROL, R y SMITS, A. (1994). "Quality judgements by patients on general practice care: a literature analysis”, Social Science \& Medicine, Vol. 38, p. 45-53.

WOLF, MH, PUTNAM, SM, JAMES, SA y STILES, WB. (1978). "The Medical Interview Satisfaction Scale: Development of a scale measure patient perceptions of physician behaviour”, Journal of Behavioral Medicine, Vol. 1, p. 391-401.

WOODRUFF, BR y GARDIAL, FS. (1996). Know your customer: New approaches to Understanding Customer Value and Satisfaction, Malden: Blackwell Business, 338 p. ISBN: 15-578-6553-1.

ZASTOWNY, TR, STRATMANN, WC, ADAMS, EH y FOX, ML. (1995). "Patient satisfaction and experience with health services and quality of care", Quality Management in Health Care, Vol. 3, p. 50-61. 